\title{
Direct space-charge calculation in ELEGANT and its application to the ILC damping ring
}

\author{
A. Xiao, M. Borland, L. Emery, Y. Wang \\ ANL, ARgonne, IL 60439, USA
}

\author{
K.Y. Ng
}

Fermilab, Batavia, IL 60510

\author{
Submitted to PAC'07 \\ Albuquerque, New Mexico \\ June 25-29, 2007
}




\title{
DIRECT SPACE-CHARGE CALCULATION IN ELEGANT AND ITS APPLICATION TO THE ILC DAMPING RING*
}

\author{
A. Xiao ${ }^{\dagger}$, M. Borland, L. Emery, Y. Wang, ANL, Argonne, IL 60439, USA \\ K. Y. Ng, FNAL, Batavia, IL 60510, USA
}

Abstract

A direct space-charge force model has been implemented in the tracking code elegant [1]. The user can simulate transverse space-charge effects by inserting spacecharge elements in the beamline at any desired position. Application to the International Linear Collider damping ring is presented in this paper. We simulated beam under equilibrium conditions, as well as the entire damping cycle from injection to extraction. Results show that beam halo is generated due to space-charge effects. This would be a significant concern for the ILC damping ring and a detailed follow-up study is needed.

\section{INTRODUCTION}

Individual particles inside an electron bunch experience direct electric and magnetic forces from all other particles. As an electron is performing synchrotron motion, the space-charge tune shifts it experiences oscillate. At the center of the bunch, these space-charge tune shifts reach the maximum values [2]

$$
\Delta \nu_{(x, y), s c}=-\frac{N_{b} r_{e}}{2 \pi \gamma^{3} \beta^{2} B}\left\langle\frac{\beta_{(x, y)}}{\sigma_{(x, y)}\left(\sigma_{x}+\sigma_{y}\right)}\right\rangle,
$$

where $N_{b}$ is the particle number in the bunch; $r_{e}$ is the classical electron radius; $B=\sqrt{2 \pi} \sigma_{z} /(2 \pi R)$ is the bunching factor; $\gamma, \beta$ are the Lorentz factors; $\beta_{(x, y)}$ are the betatron functions; and $\sigma_{x, y, z}$ are rms beam sizes in each direction. Thus the betatron tunes are constantly modulated. The results are unfavorable synchro-betatron resonances and phase space structures.

Applying Equation 1 to the International Linear Collider damping ring lattice design gives a fairly large vertical tune shift due to the large circumference-to-energy ratio, and low vertical emittance. For the OCS design [3], we get $\Delta \nu_{y} \approx-0.08$ [4]. A theoretical analysis [5] shows that $\Delta \nu_{y}<-0.1$ will cause a significant lifetime problem. Other studies $[6,7,8]$ show concerns with vertical emittance growth due to nonlinear resonance effects.

Starting from Bassetti's formula [9], we first derived the linear and nonlinear space-charge force for a Gaussian bunch. This force can be computed and applied to simulation particles through a new element, SCMULT, in elegant. The number of SCMULT elements per turn and their locations in the beamline are specified by the user, depending on the problem to be solved. This feature allows

\footnotetext{
${ }^{*}$ Work supported by the U.S. Department of Energy, Office of Science, Office of Basic Energy Sciences, under Contract No. DE-AC02$06 \mathrm{CH} 11357$.

†xiaoam@aps.anl.gov
}

greater flexibility in the simulations, since changing of optics is no longer needed and space-charge kicks can be put anywhere. The kick strength is adjusted dynamically according to the instantaneous beam parameters. The user can also choose between linear and nonlinear models. This is a self-consistent simulation and can work together with other features provided by elegant, such as synchrotron radiation damping and quantum excitation.

We applied our code to the ILC damping ring lattice [10] in the equilibrium condition. A start-to-end simulation of an entire damping cycle with radiation effects was also performed. Results show that beam halo was generated due to the space-charge effects, indicating a further detailed simulation study is needed.

\section{TRANSVERSE SPACE-CHARGE FORCE}

For a tri-Gaussian distribution bunch, the electric potential for a particle of charge $e$ at location $x, y, z$ is given by the Bassetti formula $[9,11]$

$$
U_{x, y, z}=\frac{e N_{b}}{4 \pi^{\frac{3}{2}} \epsilon_{0}} \int_{0}^{\infty} d t \frac{\exp \left[-\frac{x^{2}}{2 \sigma_{x}^{2}+t}-\frac{y^{2}}{2 \sigma_{y}^{2}+t}-\frac{z^{2}}{2 \sigma_{z}^{2}+t}\right]}{\sqrt{\left(2 \sigma_{x}^{2}+t\right)\left(2 \sigma_{y}^{2}+t\right)\left(2 \sigma_{z}^{2}+t\right)}}
$$

where $N_{b}$ is the number of particles in the bunch and $\sigma_{(x, y, z)}$ are the rms beam sizes. To include the magnetic part of the force, we need to add a $\gamma^{2}$ in the denominator. There is no closed form for this integral.

\section{Linear Approximation}

When incorporating space-charge force into a code, it will be useful if there is an option for including only the linear part. An electron bunch length is usually very much longer than the transverse size. We can therefore make the replacement

$$
2 \sigma_{z}^{2}+t \longrightarrow 2 \sigma_{z}^{2}
$$

From $F=-e \vec{\nabla} U$, we have:

$$
F_{\xi, \text { linear }}=\frac{e^{2} \xi}{2 \pi \epsilon_{0} \gamma^{2}} \frac{N_{b} e^{-s^{2} /\left(2 \sigma_{z}^{2}\right)}}{\sqrt{2 \pi} \sigma_{z}} \frac{1}{\sigma_{\xi}\left(\sigma_{x}+\sigma_{y}\right)},
$$

where $\xi$ stands for $x$ or $y$.

\section{Nonlinear Space-Charge Force}

The equations of motion in the transverse planes can be written as

$$
\xi^{\prime \prime}+K_{\xi} \xi=-\frac{e}{\gamma m v^{2}} \frac{\partial U}{\partial \xi},
$$


where $K_{\xi} \xi$ is the restoring force, $m$ is the electron mass, and $v=\beta c$ is the particle's velocity. Integrating over a length $L$ of the orbit where the beam size does not change much, we obtain the changes in $\xi^{\prime}$ due to space-charge effects:

$$
\Delta \xi_{s c}^{\prime}=-\frac{e L}{\gamma m v^{2}} \frac{\partial U}{\partial \xi} .
$$

Introducing new variables

$$
\begin{gathered}
a=\frac{x}{\sqrt{2\left(\sigma_{x}^{2}-\sigma_{y}^{2}\right)}}, \quad b=\frac{y}{\sqrt{2\left(\sigma_{x}^{2}-\sigma_{y}^{2}\right)}}, \\
k^{2}=\frac{2 \sigma_{y}^{2}+t}{2 \sigma_{x}^{2}+t}, \quad \text { and } r=\frac{\sigma_{y}}{\sigma_{x}},
\end{gathered}
$$

we obtain

$$
\begin{gathered}
\Delta x^{\prime}=K_{s c} \frac{x}{\sigma_{x}^{2}-\sigma_{y}^{2}} \int_{r}^{1} d k e^{-a^{2}\left(1-k^{2}\right)-b^{2}\left(\frac{1}{k^{2}}-1\right)}, \\
\Delta y^{\prime}=K_{s c} \frac{y}{\sigma_{x}^{2}-\sigma_{y}^{2}} \int_{r}^{1} \frac{d k}{k^{2}} e^{-a^{2}\left(1-k^{2}\right)-b^{2}\left(\frac{1}{k^{2}}-1\right)},
\end{gathered}
$$

where

$$
K_{s c}=\frac{2 N_{b} r_{e}}{\gamma^{3} \beta^{2}} \frac{L e^{-z^{2} /\left(2 \sigma_{z}^{2}\right)}}{\sqrt{2 \pi} \sigma_{z}} .
$$

The changes in the horizontal and vertical divergences can be combined as a complex variable

$$
\Delta x^{\prime}-i \Delta y^{\prime}=K_{s c} \frac{2 e^{-(a+j b)^{2}}}{\sqrt{2\left(\sigma_{x}^{2}-\sigma_{y}^{2}\right)}} \int_{a r+\frac{i b}{r}}^{a+i b} e^{\zeta^{2}} d \zeta
$$

with

$$
\zeta=a k+i \frac{b}{k} .
$$

From the definition of the complex error function

$$
w(z)=e^{-z^{2}}\left[1+\frac{2 i}{\sqrt{\pi}} \int_{0}^{z} e^{\zeta^{2}} d \zeta\right]
$$

we arrive at

$$
\begin{aligned}
\Delta x^{\prime}-i \Delta y^{\prime} & =-i \frac{K_{s c} \sqrt{\pi}}{\sqrt{2\left(\sigma_{x}^{2}-\sigma_{y}^{2}\right)}} \\
& {\left[w(a+i b)-e^{-B} w\left(a r+i \frac{b}{r}\right)\right] }
\end{aligned}
$$

with

$$
B=a^{2}\left(1-r^{2}\right)+b^{2}\left(\frac{1}{r^{2}}-1\right)
$$

\section{IMPLEMENTATION IN ELEGANT}

Equations (3) and (9) have been used in elegant for simulating space-charge effects. Since space-charge forces exist everywhere along the beamline, a detailed simulation would be very time consuming, and for some problems it may not be needed. Hence, the space-charge force is simulated as discrete kicks along the beam orbit. We define a special element SCMULT to carry out this task.

First the user has to tell elegant where to apply SCMULT kicks. The traditional way is to do this in the lattice file, but this is cumbersome and the user cannot easily change the number and location of the kicks. While this method is supported by elegant, the user can also simply ask elegant to insert SCMULT elements at desired locations using a command. An example of a command that will insert an SCMULT after every $20^{\text {th }}$ quadrupole is shown in Table 1.

Table 1: Example Command for Space-Charge Tracking

\begin{tabular}{|ll|}
\hline \&insert_sceffects & \\
& name $=*$, \\
& type $=$ "*QUAD $*$, \\
& element_prefix $=$ "MYSC" \\
& skip $=20$, \\
& vertical $=1$, \\
& horizontal $=1$, \\
& nonlinear $=1$, \\
\&end & \\
\hline
\end{tabular}

This feature makes for very flexible simulations. For example, one can use one kick per turn for quick simulations, use a kick after each element for very detailed modeling, or something in between. Using the insert_sceffects command, the user simply specifies where to insert the space-charge kicks, and the code will take care of the details. The kick strength is adjusted automatically according to local beam parameters and the distance between the SCMULT kicks.

Equation 3 is simple and can be implemented easily. Equation 9 becomes ill-defined when $\sigma_{x}=\sigma_{y}$. To handle this, the code calculates two points $\left(\sigma_{x}=\sigma_{y} \pm \epsilon\right)$ around this divergent point and averages the results. During testing, we found that very small $x$ and $y$ could cause the complex error function $w$ to overflow. To overcome this difficulty and speed up the code for nonlinear effects calculations, we use the linear force instead of the nonlinear force when $|x| \leq \sigma_{x}$ and $|y| \leq \sigma_{y}$. This criterion is a somewhat arbitrary compromise between simulation accuracy and simulation speed, and has not been optimized. However, since the kick is applied in discrete locations rather than continuously, we believe that such an approximation is justified. 


\section{APPLICATION TO ILC DAMPING RING}

A typical bunch in the ILC damping ring has beam intensity of $N_{b}=2 \times 10^{10}$ and rms bunch length $\sigma_{z}=6 \mathrm{~mm}$. The calculated linear space-charge tune shift for the OCS design is $\Delta \nu_{y} \approx-0.08$ [4]. This large tune shift could cause emittance growth due to crossing of resonance lines.

We simulated the emittance growth rate for different bunch lengths using both the linear and nonlinear models. Ten thousand particles initialized for equilibrium emittances were tracked for 1000 turns. SCMULT was added after each physical element, meaning 13224 SCMULTs were inserted into the beamline. The lattice has $\nu_{x}=$ 52.38 , and $\nu_{y}=49.25$. For $\sigma_{z}=6 \mathrm{~mm}, \nu_{s}=0.096$; and $\sigma_{z}=9 \mathrm{~mm}, \nu_{s}=0.064$. The parallel version of elegant [12] was used for fast tracking. The results are shown in Figure 1. With nonlinear space-charge effects included in our simulation, the emittance growth rate becomes smaller, which is what we expected from physical understanding. A longer bunch also results in a smaller emittance growth rate.
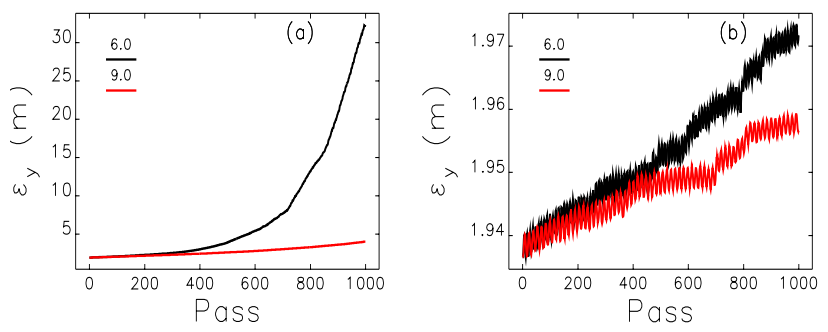

Figure 1: Emittance growth from equilibrium at different bunch lengths: (a) linear force model. (b) nonlinear force model.

Figure 2 shows a simulation of the positron beam from injection to extraction, including radiation damping and quantum excitation. The simulation indicates that a beam halo is formed due to space-charge effects and quantum excitation.

\section{CONCLUSION}

From Bassetti's formula, we derived a closed form for linear and nonlinear directed space-charge forces. The forces are applied to particles through a special element SCMULT that was added to elegant. This element can be put into a beamline directly or can be added automatically and flexibly through a command. The kick strength is adjusted automatically inside the code according to the local beam parameters. The user can choose either the linear or nonlinear model for their problem. An ILC damping ring example was given, indicating formation of beam halo at the end of the damping cycle. A more detailed simulation is needed to confirm these results. A longer bunch length (e.g., $9 \mathrm{~mm}$ ) was shown to reduce the space-charge effects.
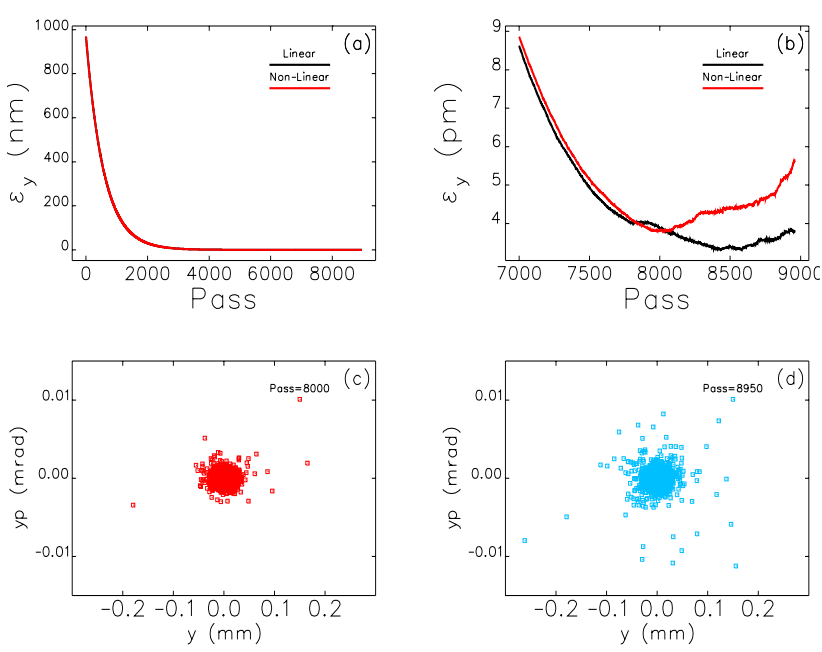

Figure 2: ILC damping ring positron beam emittance evaluation of entire damping cycle ( $\sigma_{z}=6 \mathrm{~mm}, 8962$ turns): (a) entire cycle, (b) zoom in at the end of damping cycle, (c) particles phase space at Pass $=8000$, (d) particles phase space at Pass $=8950$ (before ejection, beam halo is formed).

\section{REFERENCES}

[1] M. Borland, Advanced Photon Source LS-287, September 2000.

[2] B. Zotter, in Handbook of Accelerator Physics and Engineering, section 2.5.3, World Scientific, 1999.

[3] A. Xiao and L. Emery, "Characterization of a 6-km damping ring for the International Linear Collider," PAC-2005RPPP051.

[4] A. Wolski, J. Gao, S. Guiducci et. al, LBNL59449/Cockroft-06-04, 2006

[5] J. Gao, DESY-TESLA-2003-12, 2003.

[6] W. Decking and R. Brinkmann, DESY-M-00-04T, 2000.

[7] M. Venturini, K. Oide and A. Wolski, LBNL-59509, 2006.

[8] K. Y. Ng, NIM A 561, 276 (2006).

[9] M. Bassetti and G. A. Erskine, CERN-ISR-TH/80-06, 1980.

[10] A. Xiao, L. Emery, Argonne National Laboratory, to be published at PAC07.

[11] K. Takayama, Lett. Nuovo Cim. 34, 190 (1982).

[12] Y. Wang and M. Borland, Argonne National Laboratory, to be published at PAC07. 